SHOCK WAVES - THE TRIGGER MECHANISM OF SIO MASERS IN CIRCUMSTELLAR

ENVELOPES OF COOL GIANTS AND SUPERGIANTS

\author{
Astrid Heske \\ Hamburger Sternwarte \\ Gojenbergsweg 112 \\ 2050 Hamburg 80, F.R.G.
}

Circumstellar envelopes of cool giants and supergiants are composed of different parts which can be observed through characteristic spectral features. One is Sio-maseremission which is typically found in Miras and some semi-regular variable supergiants. These masers are thought to originate in those parts of the upper atmosphere or lower circumstellar envelope where still no mass-loss takes place. Location and trigger mechanism was still not definitely clear.

A well defined sample of about 50 cool giants and supergiants being selected by their high (I-K)-value and covering a wide range of spectral types (G8-M8) was observed, among other spectral features, in the H $\alpha-1$ ine (1.4m CAT, La Silla (Chile); $2.2 \mathrm{~m}$ Calar Alto (Spain)) and the SiO $(J=1-0)-l i n e$ (10om-telescope in Effelsberg). The resolution was $0.03 \AA$ or $1.5 \mathrm{kms}^{-1}$ and $0.7 \mathrm{kms}^{-1}$ respectively.

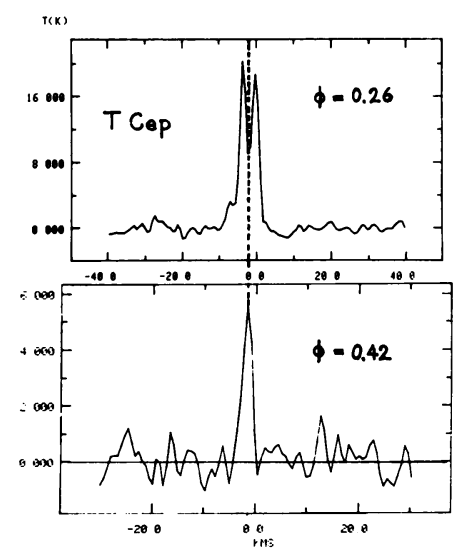

Fig.1
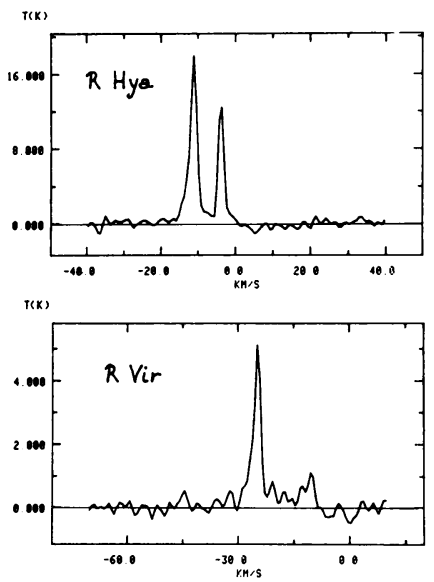

Fig. 2

Figures 1 and 2 show three examples of double-peaked maser profiles. As these profiles look similar to most of the OH-maser profiles and in $T$ Cep the single peak (observed at phase 0.42) falls exactly in the 
centre of the double-peaked profile (observed at phase 0.26) this might be taken as evidence that in these examples rear and front of a shell-type outflow of matter where Sio-masers are located is observed. Thus half of the velocity separation may be assigned to the outflow velocity. This value was found to be proportional to the ratio of the blue- to the redshifted peak intensity, $I_{\text {blue }} / I_{\text {red }}$. It can be due to a geometrical effect, the star obscuring part of the rear emitting area. If radial amplification of the masers and the peak flux being proportional to the emitting area provided, the intensity ratio $I_{\text {blue }} / I_{\text {red }}$ thus is proportional to $1 / \mathrm{R}^{2}$ as the rear emitting area grows with distance $\mathrm{R}$ from star. The Sio-masers are though found to be located between 2 and 6 stellar radii $\left(0.3 \ldots 1 * 10^{14} \mathrm{~cm}\right)$ moving out with velocities between 8 and $1 \mathrm{kms}^{-1}$.

From the whole sample comparison of the $\mathrm{H} \alpha$ - and SiO-spectra led to the correlations that SiO-masers are only observed when the giant or supergiant shows variable $\mathrm{H} \alpha$-emission and when the star is cooler than $2500 \mathrm{~K}$, if it is a giant. In supergiants SiO-maser seem to occur also at earlier types (e.g. $\mu$ Cep, M2Ib).

The observational results show evidence for Sio-masers being triggered by shock waves where Ho-emission originate. The masers may then be located in the post shock material several stellar radii above the star, moving with velocities less than $8 \mathrm{kms}^{-1}$. This is consistent with the velocity field of Mira-atmospheres derived by Willson and Bowen (1986). Their calculated density distribution implies that the particle density can still be high enough ( $\hat{N} 10^{12} \mathrm{~cm}^{-3}$ ) at several stellar radii for SiO-masers to be excited (Elitzur 1980). Shock waves as triggers for Sio-masers imply that they could be collisionally pumped as the kinetic temperature rises immensely in the shock front. The more complex maser profiles would also fit in this picture as it should be taken into account that during one cycle more than one shock wave may be expelled, and that not in every giant or supergiant the rear emitting region may be observed.

\section{References :}

M. Elitzur 1980, Astrophys. J. 240, 553

L.A. Willson and G.W. Bowen 1986, Third Trieste Workshop on the Relationship between Chromospheric/Coronal Heating and Mass-Loss; eds. R. Stalio and J. Zirker, pp. 127 